\title{
Quantitative structure-property relationships of electroluminescent materials: Artificial neural networks and support vector machines to predict electroluminescence of organic molecules
}

\author{
ALANA FERNANDES GOLIN and RICARDO STEFANI* \\ Laboratório de Estudos de Materiais (LEMAT), Instituto de Ciências Exatas e da Terra, Av. Governador Jaime Campos \\ 6390, Campus Universitário do Araguaia, Universidade Federal de Mato Grosso, 78600-00 Barra do Garças - MT. Brazil
}

MS received 13 February 2012; revised 13 December 2012

\begin{abstract}
Electroluminescent compounds are extensively used as materials for application in OLED. In order to understand the chemical features related to electroluminescence of such compounds, QSPR study based on neural network model and support vector machine was developed on a series of organic compounds commonly used in OLED development. Radial-basis function-SVM model was able to predict the electroluminescence with good accuracy $(R=\mathbf{0 . 9 0})$. Moreover, RMSE of support vector machine model is approximately half of RMSE observed for artificial neural networks model, which is significant from the point of view of model precision, as the dataset is very small. Thus, support vector machine is a good method to build QSPR models to predict the electroluminescence of materials when applied to small datasets. It was observed that descriptors related to chemical bonding and electronic structure are highly correlated with electroluminescence properties. The obtained results can help in understating the structural features related to the electroluminescence, and supporting the development of new electroluminescent materials.
\end{abstract}

Keywords. QSPR; neural networks; SVM; electroluminescence; OLED; organic materials.

\section{Introduction}

Electroluminescent materials (EL) are among the most promising modern materials with a wide range of technology applications (Xue and Luo 2003; So et al 2009). One of the most promising EL applications, is the design and fabrication of organic light-emitting diodes (OLEDs) (Akcelrud 2003). OLEDs have demonstrated manufacturing and market potential in small and medium device applications. Thus, OLED can become one of the mainstream display technologies, competing directly with LCD (liquid crystal display) technology (Wen et al 2005). For high-quality OLED displays, highly efficient and low-cost electroluminescent materials are of great importance, since, to gain market share over LCD displays, OLED devices need to be efficient and to have low prices to the final costumer. Many pyran-containing, polyaromatic hydrocarbons (PAH) and porphyrin type compounds are used in OLED fabrication and these compounds may be polymeric itself or used as a dopant to allow thinfilms to become electroluminescent (Mi et al 2002). Understanding of the physical and chemical features related to the electroluminescence of such materials, can help in the design and development of new chemical compounds with improved electroluminescence features. In order to develop new organic compounds that can be used in OLED applications, computational methods, such as quantitative-structure

\footnotetext{
*Author for correspondence (rstefani@ufmt.br)
}

properties relationships (QSPR) have emerged as a fast and reliable method to predict and study physical-chemical properties of materials.

Quantitative-structure properties relationships (QSPR) models can be used to predict with good accuracy, key physical and chemical features from chemical compounds. QSPR methods are based on the existing correlation between groups of mathematical values (descriptors), representing certain features of a chemical structure and a target chemical property. The advantage of QSPR model is that it is based solely on the knowledge of chemical structure and it requires no additional experimental data and once the correlation is established, it can be used for the prediction of properties of new compounds that have not been prepared (Yu et al 2008). Thus, QSPR models can be used to assist material design, since one can predict the properties of a certain material before its synthesis. As the development of new materials involves extensive experimental work, the ability to predict the properties of materials is of great value, because, it provides a guide to the development process and speeds up the development cycle, allowing time and reagent saving ( $\mathrm{Yu}$ et al 2008). Thus, many research groups have been developing QSPR models in order to assist material discovery and design (Morril and Byrd 2008; Taherpour 2009; Fourches et al 2010; Yu 2010). The advantage of using QSPR models over traditional computational methods is that description calculation is quite easy and requires little computation time. 
A quantitative structure-properties relationships (QSPR) study has been developed with the use of artificial neural networks (ANN) and support vector machines (SVM) in order to understand the chemical features related to the electroluminescence of a series of commonly used red dopants and nondopants used in OLED development (Chen 2004). The performance of support vector machine models was compared to the performance of artificial neural networks.

\section{Methodology}

\subsection{Dataset}

The electroluminescence data for 18 compounds (figure 1) were selected from the literature (Chen 2004) and its electroluminescence data $\left(\lambda_{\operatorname{maxEL}}\right)$ is summarized in table 1 . The free software ChemSketch 12.0 was used to draw 2-D representation of the molecules. As many of the computed descriptors depend explicitly on 3-D structures of the molecules, 3-D structures of the selected compounds were calculated using E-Corina (Tetko et al 2005).

\subsection{Descriptor calculation and selection}

In QSPR development, descriptor calculation and selection is a critical step towards a good predictive model. After 3D structure optimization, a total of 1668 physical-chemical descriptors were generated with E-DRAGON (Tetko et al 2005). In order to select the most relevant descriptors to electroluminescence, constant and near-constant descriptors were eliminated, as such descriptors are not significant and do not affect any final model. A variance-covariance matrix was calculated for all descriptors and one of two descriptors with pair wise correlation greater than $0.9(R>0.9)$ was eliminated. Thus, 1201 descriptors remained in the dataset. The final selection was made with a genetic algorithm feature selection performed with WEKA 3.4 Software (Hall et al 2009). The following procedure was applied to feature selection: - $\log$ of the maximum wavelength of electroluminescence value ( $\left.\lambda_{\operatorname{maxEL}}\right)$ (table 1 ) of each compound was calculated and set as the dependent variable, while the remaining descriptors were set as independent variables. Then, the genetic algorithm parameters were adjusted to allow a cross-over probability of 0.8 and the maximum generations and population to 100 . After run, 25 statistically significant descriptors $(F$-value $>5.000$ and $p<0.05)$ remained in the final selection (table 2). These descriptors were further used to develop artificial neural networks and support vector machines QSPR models.

\subsection{Artificial neural network model development}

To predict electroluminescence, a multi-layer perceptron (MLP) feed-forward neural network with back propagation of errors was developed. In a multi-layer perceptron (MLP) network, the individual processing units are known as perceptrons and they are usually arranged into three layers: input, hidden and output (Han et al 2005). The number of neurons in the input and output layers is determined by the number of independent, in this case the chemical features, and by the number of dependent variables, in this study, the electroluminescence. The software WEKA (Hall et al 2009) was used to develop artificial neural networks models that could predict electroluminescence with good accuracy. It generated five artificial neural networks models, using feedforward with back propagation of errors and radial-basis function kernels. The performance of each generated model was evaluated with standard statistical procedures and crossvalidation. All artificial neural networks models were validated with 10-fold leave-one-out cross-validation (LOO-cv). Leave-one-out cross-validation is a validation method that uses a single observation from an original sample as a validation data and the remaining observations as a training data (Jun et al 2011). In a 10-fold leave-one-out crossvalidation, this is repeated until 10 observations in the samples are used as validation data. It is difficult to divide small datasets into train and test sets, therefore, cross-validation is an accurate way to validate any QSPR model developed with small datasets. All cross-validation data were used to calculate the correlation coefficient, the mean absolute error, error mean and the standard deviation of errors for each generated model.

\subsection{Support vector machine model development}

Support vector machine (Smola and Schölkopf 2004) is a new method of machine learning that has been successfully applied in chemistry and materials science. The main advantage of support vector machine is that it has a few tunable parameters, such as the capacity constant, the control to the complexity of functions and the type of kernel function used for transforming the original input space into a high dimensional feature space (Xu et al 2011).

As stated before, one of the aims of the current study is to compare artificial neural networks performance with the performance of support vector machines. For this reason, QSPR models based on support vector machines were developed using the same software (WEKA) that was used for the development of QSPR artificial neural networks models. In this study, several support vector machine models with radialbasis function and sigmoid kernel were developed and the kernel parameters $C, v$ and $\gamma$ were adjusted in each experiment, while the parameter $\varepsilon$ was fixed to $0 \cdot 01$. The parameter $\varepsilon$ is important because it can tune the generalization capacity of each model. All models were cross-validated with the same method used to cross-validate artificial neural networks models.

\section{Results and discussion}

In table 2, all significant descriptors to electroluminescence are listed. It is important to note that all selected features are highly related with chemical bonding, molecular volume 
<smiles>COc1c(/C=C/C2=CC(=C(C#N)C=N)C=C(C(C)C)O2)cc2c3c1C(C)(C)CCN3CCC2(C)C</smiles><smiles>CC1=CC(=C(C#N)C#N)C=C(/C=C/c2cc3c4c(c2)CCCN4CCC3)O1</smiles><smiles>N#CCC#N</smiles><smiles></smiles><smiles>CC(C)C1=CC(C(=C=N)C#N)=CC(/C=C/c2cc3c4c(c2)C(C)(C)CCN4CCC3(C)C)O1</smiles><smiles>C=CC1=CC(=C(C#N)C#N)C=C(/C=C/c2ccc(N(CCCC)CCCC)cc2)O1</smiles><smiles></smiles><smiles>CN(C)c1ccc(/C=C/C2=CC(=C(C#N)C#N)CC(C)(C)C2)cc1</smiles><smiles>CCN(CC)c1ccc2c(c1)OC(/C=C/C=C(C#N)C#N)C(c1cccs1)=C2</smiles>

12<smiles>c1ccc(-c2c3cc4ccccc4cc3c(-c3ccccc3)c3cc4ccccc4cc23)cc1</smiles>

16<smiles></smiles>

17<smiles>CNc1ccc(C)cc1</smiles>

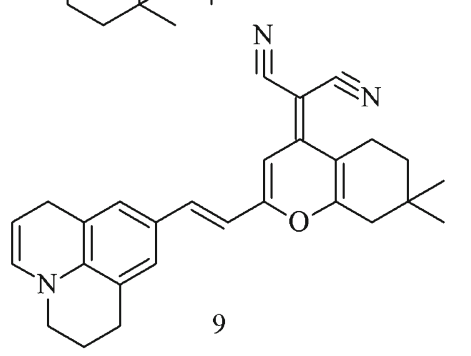<smiles>C=CCCNCCCC</smiles><smiles></smiles><smiles>CN(C)c1ccc(C=NC(C#N)=C(C#N)N=Cc2ccc(N(C)C)cc2)cc1</smiles>

18

Figure 1. Chemical structures of 18 electroluminescent compounds selected to ANN and SVM developments.

and electronic structure of the studied compounds. The first three significant descriptors are $R 7 p+, R 7 m+$ and $R 8 e+($ table 2 ), which are a type of GETAWAY (geometry, topology and atom weights assembly) descriptor weighted, respectively by atomic polarizabilities, molar mass and electronegativity (Todeschini and Consonni 2009). These 
descriptors are calculated from the leverage matrix obtained by the centred atomic coordinates and are closely related to molecule geometry and the position of substituents in

Table 1. Experimental EL performance values expressed in $\mathrm{nm}$ $\left(\lambda_{\operatorname{maxEL}}\right)$ and normalization $(-\log )$ to QSPR model development.

\begin{tabular}{lcc}
\hline & \multicolumn{2}{c}{ Compound } \\
Sl. No. & $\lambda$ maxEL $(\mathrm{nm})$ & $-\log \left(\lambda_{\operatorname{maxEL}}\right)$ \\
\hline 1 & 645 & $-2 \cdot 81291$ \\
2 & 774 & $-2 \cdot 80884$ \\
3 & 630 & $-2 \cdot 79939$ \\
4 & 624 & $-2 \cdot 79518$ \\
5 & 645 & $-2 \cdot 81291$ \\
6 & 660 & $-2 \cdot 81954$ \\
7 & 670 & $-2 \cdot 82607$ \\
8 & 660 & $-2 \cdot 81954$ \\
9 & 680 & $-2 \cdot 83250$ \\
10 & 670 & $-2 \cdot 82607$ \\
11 & 653 & $-2 \cdot 81491$ \\
12 & 629 & $-2 \cdot 79934$ \\
13 & 650 & $-2 \cdot 81291$ \\
14 & 622 & $-2 \cdot 79379$ \\
15 & 624 & $-2 \cdot 79518$ \\
16 & 625 & $-2 \cdot 79588$ \\
17 & 616 & $-2 \cdot 78958$ \\
18 & 616 & $-2 \cdot 78958$ \\
\hline
\end{tabular}

the molecule. Therefore, they are suitable to describe differences in molecular properties caused by the position and alignment of substituent groups and its electronic features. The second three significant descriptors are BEHm1, BELm2 and BEHm3 (table 2). They are in the group of Burden eigenvalues index (Todeschini and Consonni 2009) descriptors, which are topological indices that are weighted by relative atomic masses. Burden indices describe molecules in a topological way. Thus, the molecule topology has influence on electroluminescence. $P 1 p$ and $P 1 m$ (table 2) are weighted holistic invariant molecular descriptors (WHIM), weighted respectively by atomic polarizability and relative atomic mass. WHIM descriptors are geometrical descriptors derived from the geometrical distance between the atoms of a molecule. These descriptors are based on statistical indices calculated on the projections of atoms along the principal axes, allowing to capture the 3-D information regarding size, shape, symmetry and atom distributions with respect to invariant reference frames. Thus, it can provide information about the correlation between the three-dimensional structure of a molecule and a certain molecule property. $K s$ (table 2) is also a WHIM descriptor, but it is weighted by atomic electrotopological state (E-STATE). The weight function can provide information on the electronic and topological state of each atom in the molecule (Todeschini and Consonni 2009). The state of each atom is a ratio of $\pi$ and lone-pair electrons over the count of $\sigma$ bonds of the considered atom in

Table 2. Statically significant selected descriptors

\begin{tabular}{|c|c|c|c|c|}
\hline $\begin{array}{l}\text { Descriptor } \\
\text { symbol }\end{array}$ & Descriptor name & $F$-value & $t$-test & $p$-value \\
\hline$R 7 p+$ & $R$ maximal autocorrelation of lag $7 /$ weighted by atomic polarizabilities & $6 \cdot 070884$ & $836 \cdot 207$ & $0 \cdot 005001$ \\
\hline$R 7 m+$ & $R$ maximal autocorrelation of lag 7/weighted by atomic masses & $5 \cdot 517095$ & $824 \cdot 289$ & $0 \cdot 008043$ \\
\hline$R 8 e+$ & $R$ maximal autocorrelation of lag $8 /$ weighted by atomic Sanderson electronegativities & $6 \cdot 353988$ & 797.765 & $0 \cdot 004851$ \\
\hline BEHm1 & Highest eigenvalue no. 1 of burden matrix/weighted by atomic masses & $5 \cdot 757409$ & $254 \cdot 122$ & $0 \cdot 006832$ \\
\hline BELm2 & Lowest eigenvalue no. 2 of burden matrix/weighted by atomic masses & $7 \cdot 765038$ & $229 \cdot 706$ & $0 \cdot 002941$ \\
\hline $\mathrm{BEHm} 3$ & Highest eigenvalue no. 3 of burden matrix/weighted by atomic masses & $5 \cdot 391306$ & $224 \cdot 527$ & $0 \cdot 007982$ \\
\hline$P 1 p$ & 1st component shape directional WHIM index/weighted by atomic polarizabilities & $5 \cdot 891804$ & $147 \cdot 394$ & $0 \cdot 005626$ \\
\hline$P 1 m$ & 1st component shape directional WHIM index/weighted by atomic masses & $6 \cdot 044614$ & $140 \cdot 618$ & $0 \cdot 005087$ \\
\hline HOMA & Harmonic oscillator model of aromaticity index & $5 \cdot 861169$ & $105 \cdot 839$ & $0 \cdot 005742$ \\
\hline Ks & $K$ global shape index/weighted by atomic electrotopological states & $8 \cdot 535021$ & $104 \cdot 418$ & $0 \cdot 001318$ \\
\hline $\mathrm{CICO}$ & Complementary information content index (neighbourhood symmetry of 0 -order) & $6 \cdot 776535$ & $88 \cdot 190$ & $0 \cdot 003811$ \\
\hline GATS4v & Geary autocorrelation of lag 4 weighted by Van der Waals volume & $8 \cdot 318028$ & $46 \cdot 724$ & $0 \cdot 001479$ \\
\hline GATS7e & Geary autocorrelation of lag 7 weighted by Sanderson electronegativity & $6 \cdot 571426$ & $30 \cdot 832$ & $0 \cdot 003639$ \\
\hline MAXDP & Maximal electrotopological positive variation & $5 \cdot 770581$ & 21.439 & $0 \cdot 008751$ \\
\hline HTu & $\mathrm{H}$ total index/unweighted & $9 \cdot 599787$ & $20 \cdot 738$ & $0 \cdot 000774$ \\
\hline RDF095v & Radial distribution function-095/weighted by Van der Waals volume & $5 \cdot 861374$ & $11 \cdot 577$ & $0 \cdot 005832$ \\
\hline RDF060m & Radial distribution function-060/weighted by atomic masses & $5 \cdot 214013$ & $9 \cdot 780$ & $0 \cdot 009907$ \\
\hline RDF125v & Radial distribution function-125/weighted by Van der Waals volume & $8 \cdot 109778$ & 7.743 & $0 \cdot 001654$ \\
\hline RDF125p & Radial distribution function-125/weighted by polarizability & $7 \cdot 235529$ & $7 \cdot 690$ & $0 \cdot 002708$ \\
\hline RDF135v & Radial distribution function-135/weighted by Van der Waals volume & $5 \cdot 742541$ & $5 \cdot 580$ & $0 \cdot 006901$ \\
\hline RDF135p & Radial distribution function-125/weighted by polarizability & $9 \cdot 871466$ & $5 \cdot 148$ & $0 \cdot 000935$ \\
\hline RDF135u & Radial distribution function-35/unweighted & $5 \cdot 613742$ & $3 \cdot 797$ & $0 \cdot 009681$ \\
\hline RDF145e & Radial distribution function-145/weighted by Sanderson electronegativity & $6 \cdot 450780$ & 3.429 & $0 \cdot 005735$ \\
\hline RDF145u & Radial distribution function-145/unweighted & $6 \cdot 450780$ & $3 \cdot 402$ & $0 \cdot 005735$ \\
\hline RDF155u & Radial distribution function-155/unweighted & $6 \cdot 759098$ & $2 \cdot 814$ & $0 \cdot 004773$ \\
\hline
\end{tabular}


the molecular graph. Therefore, the number of $\pi$ electrons in a molecule is related to its electroluminescence. This is an expected feature, since intermolecular $\pi$-stacking can lead to aggregation in solid state, which can cause changes in electroluminescence (Chen 2004). Thus, the knowledge of the electrotopological structure can help in the design and development of new molecules which are less prone to aggregation in solid state. MAXDP (table 2) is a descriptor that represents the maximal electrotopological positive variation, confirming the great influence of electronic topology in electroluminescence. HOMA (table 2) is the harmonic oscillator model of aromatic. This is a descriptor strictly related to the degree of delocalization of $\pi$ electrons in an aromatic system and an extension of delocalization. Hence, the degree of $\pi$ electron delocalization has influence on electroluminescence. CIC0 (table 2) is an information index related to the 0 -order neighbourhood of atom and it can be considered a descriptor related to the position and pattern of substitution. $\tau$ (table 2 ) denotes $H$ total index, i.e., the total index of hydrogen atoms in the molecule. Another type of selected descriptors is 2D-autocorrelation descriptors represented by GATS4v and GATS7e (table 2) descriptors. These descriptors are weighted by molar volume and electronegativity,

Table 3. Comparison between performances of trained SVM models.

\begin{tabular}{lcccccc}
$\begin{array}{l}\text { Model } \\
\text { numbers }\end{array}$ & $\mathrm{C}$ & $v$ & $\gamma$ & Kernel & Coefficient & Correlation \\
\hline 1 & $10 \cdot 000$ & $0 \cdot 300$ & 0.001 & Sigmoid & 0.1 & 0.80584 \\
2 & $10 \cdot 000$ & 0.500 & 0.001 & Sigmoid & 0.01 & 0.22163 \\
3 & $10 \cdot 000$ & 0.500 & 0.001 & RBF & - & 0.90167 \\
4 & 5.000 & 0.500 & 0.001 & RBF & - & 0.27272 \\
\hline
\end{tabular}

respectively. Thus, molar volume and electronegativity have influence on electroluminescence. The last group of representative descriptors is the radial distribution function (RDF) descriptor type (Gasteiger et al 1996). These descriptors are 3-D descriptors that encode the molecular 3D-structure and can be unweighted or weighted by some function. In this work, most RDF descriptors (table 2) are weighted by polarizability, electronegativity and molar volume. This corroborates, along other descriptors, the influence of the electronic structure and state in electroluminescence.

Analysing the performance of each machine learning experiment, the best support vector machine and artificial neural network models have been selected to performance comparison and discussion. In table 3 , the performance of support vector machine models is given and the best support vector machine model (3) was that with radial-basis function kernel and the parameters $C=10.000, v=0.500$ and $\gamma=$ $0 \cdot 001$. The comparison between the experimental and calculated electroluminescence value is depicted in figure 2, with a correlation coefficient of 0.90167 and mean absolute error (MAE) of 0.00117. In table 4, the performance of artificial neural networks models is given and the best artificial neural networks model (2) was a network with radial-basis function kernel multilayer perceptron architecture with 25 neurons in the input layer, 5 neurons in the hidden layer and one neuron in the output layer. The artificial neural network was optimized with a train error of 0.043521 , a selection error of 0.000147 and a test error of 0.263794 . The comparison between the experimental and calculated electroluminescence value is depicted in figure 3 with a correlation coefficient of 0.80671 .

Based on the obtained results, the two methods: artificial neural networks and support vector machine were compared. The comparison between MAE, RMSE, $q^{2}$ and the regression coefficient for the two best artificial neural networks and support vector machine models are listed in table 5. The

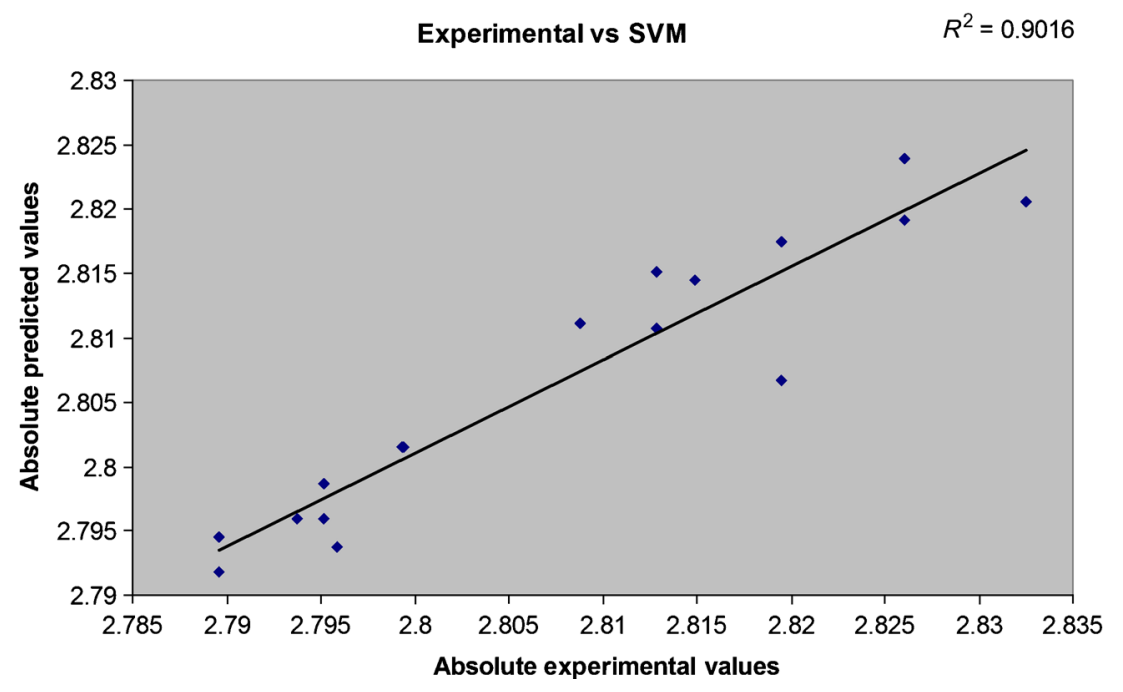

Figure 2. Plot of experimental vs calculated SVM $\left(\lambda_{\operatorname{maxEL}}\right)$ values. 


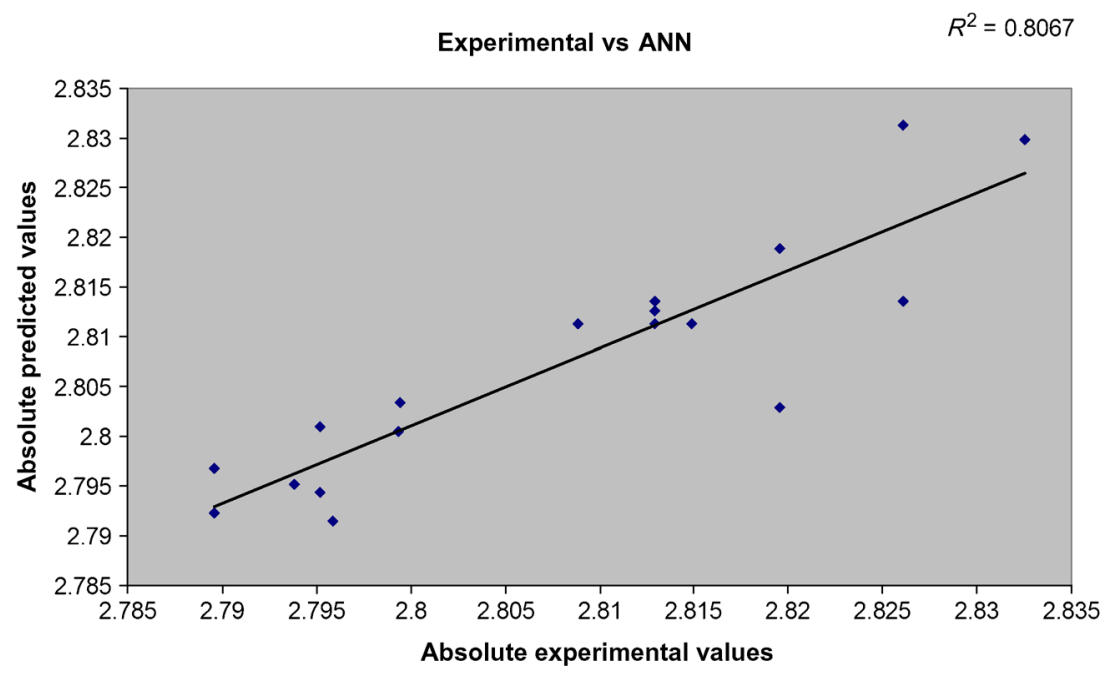

Figure 3. Plot of experimental vs calculated ANN ( $\left.\lambda_{\operatorname{maxEL}}\right)$ values.

Table 4. Comparison between performances of trained ANN models.

\begin{tabular}{|c|c|c|c|c|c|c|}
\hline Model numbers & Architecture & Train error & Select error & Test error & Kernel & Correlation \\
\hline 1 & MLP 25:5:1 & 0.073037 & $0 \cdot 000061$ & $0 \cdot 375799$ & Sigmoid & 0.75757 \\
\hline 2 & RBF 25:5:1 & $0 \cdot 043521$ & $0 \cdot 000147$ & $0 \cdot 263794$ & $\mathrm{RBF}$ & $0 \cdot 80671$ \\
\hline 3 & RBF 25:3:1 & 0.410573 & 0.062348 & 0.776414 & $\mathrm{RBF}$ & $0 \cdot 70496$ \\
\hline 4 & MLP 25:8:1 & $0 \cdot 162391$ & 0.056762 & 0.468712 & Sigmoid & 0.72583 \\
\hline
\end{tabular}

Table 5. Comparison of experimental and calculated value of electroluminescence data ( $\left.\lambda_{\text {maxEL }}\right)$ using the best ANN and SVM models and its performance.

\begin{tabular}{|c|c|c|c|}
\hline Compounds & $-\log \left(\lambda_{\operatorname{maxEL}}\right)(\operatorname{Exp})$ & SVM RBF kernel (calcd) & ANN RBF kernel (calcd) \\
\hline 1 & $-2 \cdot 81291$ & $-2 \cdot 81076$ & $-2 \cdot 81361$ \\
\hline 2 & $-2 \cdot 80884$ & $-2 \cdot 81104$ & $-2 \cdot 81122$ \\
\hline 3 & $-2 \cdot 79939$ & $-2 \cdot 80151$ & $-2 \cdot 80338$ \\
\hline 4 & $-2 \cdot 79518$ & $-2 \cdot 79872$ & $-2 \cdot 80091$ \\
\hline 5 & $-2 \cdot 81291$ & $-2 \cdot 81508$ & $-2 \cdot 81137$ \\
\hline 6 & $-2 \cdot 81954$ & $-2 \cdot 80676$ & $-2 \cdot 80291$ \\
\hline 7 & $-2 \cdot 82607$ & $-2 \cdot 82391$ & $-2 \cdot 81355$ \\
\hline 8 & $-2 \cdot 81954$ & $-2 \cdot 81738$ & $-2 \cdot 81881$ \\
\hline 9 & $-2 \cdot 83250$ & $-2 \cdot 82053$ & $-2 \cdot 82991$ \\
\hline 10 & $-2 \cdot 82607$ & $-2 \cdot 81915$ & $-2 \cdot 83122$ \\
\hline 11 & $-2 \cdot 81491$ & $-2 \cdot 81441$ & $-2 \cdot 81126$ \\
\hline 12 & $-2 \cdot 79934$ & $-2 \cdot 80151$ & $-2 \cdot 80043$ \\
\hline 13 & $-2 \cdot 81291$ & $-2 \cdot 81074$ & $-2 \cdot 81256$ \\
\hline 14 & -2.79379 & -2.79595 & $-2 \cdot 79524$ \\
\hline 15 & $-2 \cdot 79518$ & $-2 \cdot 79592$ & $-2 \cdot 79433$ \\
\hline 16 & $-2 \cdot 79588$ & $-2 \cdot 79372$ & $-2 \cdot 79152$ \\
\hline 17 & $-2 \cdot 78958$ & $-2 \cdot 79175$ & $-2 \cdot 79677$ \\
\hline 18 & $-2 \cdot 78958$ & $-2 \cdot 79455$ & $-2 \cdot 79219$ \\
\hline Mean absolute error (MAE) & - & $0 \cdot 00117$ & $0 \cdot 00395$ \\
\hline Standard deviation (SD) of error & - & $0 \cdot 00347$ & 0.00577 \\
\hline Root mean square error (RMSE) & - & $0 \cdot 03420$ & $0 \cdot 06285$ \\
\hline$q^{2}$ & & $0 \cdot 85885$ & 0.80311 \\
\hline Correlation & - & $0 \cdot 90169$ & $0 \cdot 80672$ \\
\hline
\end{tabular}


best support vector machine model $\left(R^{2}=0 \cdot 90167\right.$, MAE $=$ $0.00117, \mathrm{RMSE}=0.03420$ and $\left.q^{2}=0.85885\right)$ exhibit better performance than the best artificial neural networks model $\left(R^{2}=0.80395\right.$, MAE $=0.00395$, RMSE $=0.06285$ and $q^{2}=0.80311$ ). As the higher values of $R^{2}$ and $q^{2}$ indicate higher predictive power of the model (Shahbazikhah et al 2011), it is clear that the support vector machine model has higher predictive power than artificial neural networks model. Support vector machine regression is suitable to be applied in QSPR studies with small datasets, since it can interpret the nonlinear relationships between a molecular structure and its properties (Juna et al 2010), while artificial neural networks generally need larger datasets to exhibit good performance. Thus, artificial neural networks performance can be improved and can even be similar to the support vector machine performance if large datasets are available.

\section{Conclusions}

In this study, QSPR models to predict the electroluminescence of OLED materials were developed by using support vector machine and artificial neural networks. Since, QSPR studies is based solely on the chemical structure, the obtained results can help in the understating of the structural features related to electroluminescence, supporting the development of new electroluminescent materials. The statistical results of the radial-basis function-support vector machine model indicate a good predictive model. The difference between the observed RMSE for artificial neural networks and support vector machine models is significant from the point of view of model confidence, as the dataset is small. Thus, when applied to small datasets, Support vector machine is a good method to build QSPR models to predict the electroluminescence of chemical compounds.

\section{Acknowledgements}

The authors acknowledge the financial support from CAPES (grant for A F G) and FAPEMAT (Proc. No. 835372/2009).

\section{References}

Akcelrud L 2003 Prog. Polym. Sci. 28875

Chen C T 2004 Chem. Mater. 164389

Fourches D, Pu D, Tassa C, Weissleder R, Shaw S Y, Mumper R J, Tropsha A 2010 ACS Nano 45703

Gasteiger J, Sadowski J, Schuur J, Selzer P, Steinhauer L and Steinhauer V 1996 J. Chem. Inf. Comput. Sci. 361030

Hall M, Frank E, Holmes G, Pfahringer G, Reutmann P and Witten I H 2009 The WEKA data mining Software: An Update. SIGKDD Explorations 11

Han I-S, Han C and Chung C-B 2005 J. Appl. Polym. Sci. 95967

Jun Qi, Wei J, Sun C and Pan T 2011 Front. Earth. Sci. 5245

Juna Q, Chang-Honga S and Jiac W 2010 Proc. Env. Sci. 21429

Mi B X et al 2002 J. Mater. Chem. 121307

Morril J A and Byrd E F C 2008 J. Mol. Graph. Model 27349

Shahbazikhah P, Asadollahi-Baboli M, Khaksar R, Alamdaria R F, Zare-Shahabadic V 2011 J. Braz. Chem. Soc. 221446

Smola A J and Schölkopf B 2004 Stat. Comput. 14199

So K H, Park H-T, Shin S C, Lee S G, Lee D H, Oh H Y, Kwon S K, Kim Y-H 2009 Bull. Korean Chem. Soc. 301611

Taherpour A 2009 Chem. Phys. Lett. 483233

Tetko I V et al 2005 J. Comp. Aid. Mol. Des. 19453

Todeschini R and Consonni V 2009 Molecular descriptors for chemoinformatics (Wiley-VCH: Weinheim)

Wen S-W, Lee M-T and Chen C H 2005 J. Disp. Technol. 190

Xu J, Wang L, Wang L U, Shen X and Xu W 2011 J. Comp. Chem. 121

Xue C and Luo F T 2003 Tetrahedron 595193

Yu X 2010 Fiber Polym. 11757

Yu X, Yi B, Yu W and Wang X 2008 Chem. Papers 62623 